\title{
Nonexistence of Certain Levi Flat Hypersurfaces in Kähler Manifolds from the Viewpoint of Positive Normal Bundles
}

\author{
To the memory of Marco Brunella
}

by

\author{
Takeo OHSAWA
}

\begin{abstract}
Combining the $L^{2}$ method of solving the $\bar{\partial}$-equation with a method of Brunella in the study of Levi flat hypersurfaces as stable sets of holomorphic foliations, two nonexistence theorems are proved for Levi flat hypersurfaces extending a result of Brunella.
\end{abstract}

2010 Mathematics Subject Classification: 32V40, 32D15.

Keywords: Kähler manifold, Levi flat hypersurface, $L^{2}$ estimates for $\bar{\partial}$.

\section{Introduction}

Let $X$ be a connected complex manifold of dimension $n$ and let $M \subset X$ be a closed real hypersurface of class $C^{1} . M$ is said to be Levi flat if $M$ is foliated by $(n-1)$-dimensional complex submanifolds of $X$, or equivalently if $M$ is locally pseudoconvex from both sides (cf. [Sh] and $[\mathrm{A}]$ ).

In 2008, M. Brunella [B-1] proved: Suppose that $n \geq 3, X$ is compact and admits a Kähler metric, $M$ is a Levi flat hypersurface of class $C^{2, \alpha}, \alpha>0$, and there exists on some neighborhood $U$ of $M$ a holomorphic foliation $\mathcal{F}$ which leaves $M$ invariant. Then the normal bundle of $\mathcal{F}$ does not admit any fiber metric with positive curvature. (Concerning what happens if $n=2$, see [Oh-4, Appendix], for instance.)

This result is an extension of Lins-Neto's theorem in [L] asserting the nonexistence of real analytic Levi flat hypersurfaces in $\mathbb{C P}^{n}$, the complex projective space

Communicated by T. Mochizuki. Received April 24, 2012. Revised July 27, 2012, October 4, 2012.

T. Ohsawa: Graduate School of Mathematics, Nagoya University, Nagoya 464-8602 Japan; e-mail: ohsawa@math.nagoya-u.ac.jp 
of dimension $n$, if $n \geq 3$. In fact such a hypersurface, together with a holomorphic foliation it induces on its neighborhood, would contradict Brunella's theorem, since the positivity of the holomorphic bisectional curvature of $\mathbb{C P}^{n}$ induces on the normal bundle a fiber metric with positive curvature.

The study of Levi flat hypersurfaces is related to basic questions in the theory of foliations and dynamical systems as well as those in complex algebraic geometry and several complex variables (see also [B-2, B-3], [C-L] and [S]). In [B-1, B-2], it is remarked that [Oh-6] is somehow concerned with these studies. Indeed, in [Oh-5] preceding it, it is proved: Let $X$ be a compact Kähler manifold and let $M \subset X$ be a real analytic Levi flat hypersurface. Then there exist no $C^{\infty}$ exhaustion functions on $X-M$ whose Levi form is semipositive of rank $\geq 3$ outside a compact subset.

This gives another proof of Lins-Neto's theorem, and was used in [Oh-6] to classify the Levi flat hypersurfaces in complex tori. A classification of holomorphic foliations, possibly with singularities, was done on complex tori in [B-2] in a similar spirit.

With such background, as a continuation of [Oh-5, Oh-6], we present two results on the nonexistence of Levi flat hypersurfaces that match Brunella's viewpoint.

The first one is as follows.

Theorem 0.1. Suppose that $n \geq 3, X$ is a compact Kähler manifold, $M$ is a Levi flat hypersurface of class $C^{2, \alpha}, \alpha>0$, and there exists on some neighborhood $U$ of $M$ a holomorphic foliation $\mathcal{F}$ which leaves $M$ invariant. Then the normal bundle of $\mathcal{F}$ does not admit any fiber metric whose curvature form restricted to the tangent spaces of $\mathcal{F}$ is semipositive of rank $\geq 2$ at every point of $M$.

The proof is based on Brunella's method of constructing a strictly plurisubharmonic exhaustion function on $X-M$ under his curvature assumption, which will be reproduced in $\S 1$ with some simplification. Brunella's method has a novelty compared with the previously known ways of producing plurisubharmonic functions from distance functions, since they all exploited more or less the semipositivity of holomorphic bisectional curvature of some Kähler metric on $X$ (cf. [O], [T-1, T-2], [E], [Oh-4], [M]). Applying Brunella's method in our situation, we have on $X-M$ an exhaustion function which may not be plurisubharmonic any more, but still can lead to a contradiction. Such an argument in [Oh-5] applies Hartogs type extension theorems for analytic cohomology classes. The extension is based on a Grauert-Riemenschneider type generalization of Hodge-Kodaira-Nakano's theory of harmonic forms to certain manifolds with boundary. The principal assertion in this theory is the vanishing of some analytic cohomology classes, or the solvability of certain $\bar{\partial}$-equations in other words. In the present article, for 
the proof of Theorem 0.1, we need to apply a slight generalization of GrauertRiemenschneider's vanishing theorem on complete Kähler manifolds (cf. Theorem 2.1 ), essentially due to Takegoshi [ $\mathrm{Tg}$ ], and results of such kind obtained as corollaries of a theorem in [Oh-Tg] (cf. Theorem 2.2 and its corollaries) with respect to the $L^{2} \bar{\partial}$-cohomology groups. The main ingredient of the present article is an observation that the above mentioned "almost plurisubharmonic" exhaustion functions produced by Brunella's method are exactly what is needed for an abstract but basic harmonic representation theorem in [Oh-Tg] to hold. For other $L^{2}$ Hodge theorems which are not directly applicable here, see [Oh-2] and [Dm]. We note that the condition imposed on the rank of the curvature form is optimal (cf. [Oh-4]).

Proceeding further we shall prove

Theorem 0.2. Suppose that $X$ is a compact Kähler manifold of dimension $\geq 3$, and $M$ is a Levi flat hypersurface of class $C^{\infty}$. Then the holomorphic normal bundle of $M$ does not admit a fiber metric whose curvature form is semipositive of rank $\geq 2$ on the holomorphic tangent spaces of $M$.

Roughly speaking, Brunella's method reduces Theorem 0.2 to Theorem 0.2 of $[\mathrm{Oh}-5]$.

\section{$\S 1$. Constructing exhaustion functions from fiber metrics}

First we briefly summarize how Brunella [B-1] constructed exhaustion functions on the complements of Levi flat hypersurfaces with positive normal bundles. It will then be clear what we shall have under weaker curvature assumptions.

Recall that, given a holomorphic one-codimensional foliation $\mathcal{F}$ on an open set $U$ of a complex manifold $X$, the normal bundle of $\mathcal{F}$ denoted by $N_{\mathcal{F}}$ is defined as the quotient of the holomorphic tangent bundle $T_{U}^{1,0}$ of $U$ by the subbundle $T_{\mathcal{F}}^{1,0}$ consisting of the vectors that are tangent to $\mathcal{F}$. With respect to an open covering $\left\{U_{\alpha}\right\}_{\alpha \in I}$ of $U$, for which one has a system of locally defined closed holomorphic 1-forms $\omega_{\alpha}(\alpha \in I)$ such that $\operatorname{Ker} \omega_{\alpha} \subset T_{\mathcal{F}}^{1,0}$, the system of functions $e_{\alpha \beta}$ on $U_{\alpha} \cap U_{\beta}(\alpha, \beta \in I)$ satisfying $e_{\alpha \beta} \omega_{\beta}=\omega_{\alpha}$ is a multiplicative 1-cocycle defining the line bundle $N_{\mathcal{F}}$ with respect to the dual frames $\omega_{\alpha}^{*}$ of $\omega_{\alpha}$. Note that the system $\omega=\left\{\omega_{\alpha}\right\}$ is canonically identified with an $N_{\mathcal{F}}$-valued holomorphic 1-form. We shall call $\omega$ a defining 1 -form of $\mathcal{F}$. One may identify $\omega$ with a section of $\left(T_{U}^{1,0}\right)^{*} \otimes N_{\mathcal{F}}$ locally expressed by $\omega_{\alpha} \otimes \omega_{\alpha}^{*}$. For any $C^{2}$ Levi flat hypersurface $M$, defining 1-forms of the foliation on $M$ are defined similarly. A fiber metric of $N_{\mathcal{F}}$ is identified with a system of positive $C^{\infty}$ functions $h_{\alpha}$ on $U_{\alpha}$ satisfying $h_{\alpha}=\left|e_{\beta \alpha}\right| h_{\beta}$ on $U_{\alpha} \cap U_{\beta}$. For each vector $\zeta=\zeta_{\alpha} \omega_{\alpha}^{*}\left(\zeta_{\alpha} \in \mathbb{C}\right)$ in the fiber $N_{\mathcal{F}, x}$ of $N_{\mathcal{F}}$ at $x$, the squared length of $\zeta$ with respect to $h$ is defined as $\left|\zeta_{\alpha}\right|^{2} h_{\alpha}(x)$. The curvature form of the fiber metric 
$h=\left\{h_{\alpha}\right\}_{\alpha \in I}$ is the globally well defined $(1,1)$-form $\partial \bar{\partial} \log \left(1 / h_{\alpha}\right)$ on $U$, which will be denoted by $\Theta_{h}$. We shall regard $\Theta_{h}$ as a Hermitian form on the fibers of $T_{U}^{1,0}$ by $\Theta_{h}(\xi \wedge \eta)\left(\xi, \eta \in T_{U, x}^{1,0}, x \in U\right)$.

Proposition 1.1. Let $X$ be a complex manifold, and let $M$ be a compact Levi flat hypersurface of class $C^{2, \alpha}(\alpha>0)$ in $X$ such that there exists a neighborhood $U \supset M$ and a holomorphic foliation $\mathcal{F}$ of codimension one on $U$ such that $T_{\mathcal{F}}^{1,0} \mid M$ is contained in the complexified tangent bundle of $M$. Suppose that $N_{\mathcal{F}}$ admits a fiber metric with positive curvature. Then there exist a neighborhood $V$ of $M$ in $U$ and a $C^{\infty}$ function $\varphi$ on $X-M$ such that $\partial \bar{\partial} \varphi>0$ on $V-M$ and $\lim _{x \rightarrow M} \varphi(x)=+\infty$.

Proof. By shrinking $U$ and taking its double cover if necessary, we fix a real valued $C^{2, \alpha}$ function $\varrho$ on $U$ such that $\varrho^{-1}(0)=M$ and $d \varrho$ vanishes nowhere on $M$. Let $W_{\gamma}(\gamma \in J)$ be a family of local coordinate neighborhoods in $X$ such that $M$ is contained in the union of $W_{\gamma}$ and there exists for each $\gamma$ a real valued $C^{2, \alpha}$ function $u_{\gamma}$ on $M \cap W_{\gamma}$ satisfying $\operatorname{Ker}\left(d u_{\gamma}\right)_{x}=T_{\mathcal{F}, x}$ for every $x \in W_{\gamma} \cap M$. Let $\widetilde{u}_{\gamma}$ be a $C^{2, \alpha}$ extension of $u_{\gamma}$ to the set $W_{\gamma} \cap\{\varrho \leq 0\}$ such that $\bar{\partial} \widetilde{u}_{\gamma}=0$ on $W_{\gamma} \cap\{\varrho<0\}$. Existence of $\widetilde{u}_{\gamma}$ can be seen by the classical boundary regularity property of conformal mappings on the unit disc in $\mathbb{C}$ as in [B-1] (cf. [P]), combined with the holomorphicity of the foliation $\mathcal{F}$.

Let $\omega$ be a defining 1-form of $\mathcal{F}$ on a neighborhood of $M$. We may assume that $\omega=\left\{\omega_{\gamma}\right\}_{\gamma \in J}$, where $\omega_{\gamma}$ are defined on $W_{\gamma}$. Let $h=\left\{h_{\gamma}\right\}$ be a fiber metric of $N_{\mathcal{F}}$ on a neighborhood of $M$ such that

$$
h_{\delta}=\left|\omega_{\gamma} / \omega_{\delta}\right|^{2} h_{\gamma}
$$

on $W_{\gamma} \cap W_{\delta}$.

By the positivity assumption on the bundle $N_{\mathcal{F}}$, one may assume that $\log \left(1 / h_{\gamma}\right)$ are strictly plurisubharmonic.

Since $\omega_{\gamma}=e_{\gamma} d \widetilde{u}_{\gamma}$ on $W_{\gamma} \cap\{\varrho \leq 0\}$ for some $C^{1, \alpha}$ function $e_{\gamma}$ which is nowhere vanishing on $W_{\gamma} \cap\{\varrho \leq 0\}$ and holomorphic on $W_{\gamma} \cap\{\varrho<0\}$, one can infer from (1.1) the following relation between $h_{\gamma}$ and $\left\{\widetilde{u}_{\gamma}\right\}$ :

$$
h_{\gamma}\left|e_{\gamma}\right|^{2}\left(\operatorname{Im} \widetilde{u}_{\gamma}\right)^{2}-h_{\delta}\left|e_{\delta}\right|^{2}\left(\operatorname{Im} \widetilde{u}_{\delta}\right)^{2}=O\left(\varrho^{3}\right) \quad \text { on } W_{\gamma} \cap W_{\delta} \cap\{\varrho \leq 0\}
$$

for any $\gamma, \delta \in J$. Here $O\left(\varrho^{3}\right)$ stands for any function which is bounded from above by const $\cdot|\varrho|^{3}$.

From (1.2) it is obvious that there exists a $C^{1, \alpha}$ function $v$ on the set $\{\varrho \leq 0\}$ satisfying the equality $v=h_{\gamma}\left|e_{\gamma}\right|^{2}\left(\operatorname{Im} \widetilde{u}_{\gamma}\right)^{2}+O\left(\varrho^{3}\right)$ on $W_{\gamma} \cap\{\varrho \leq 0\}$ for each $\gamma$. Then, combining the strict plurisubharmonicity of $\log \left(1 / h_{\gamma}\right)$ with (1.3) and the holomorphicity of $e_{\gamma}$ on $W_{\gamma} \cap\{\varrho<0\}$, it is easy to see that the function $-\log v$ is 
strictly plurisubharmonic on $V \cap\{\varrho<0\}$ for a sufficiently small neighborhood $V$ of $M$.

A desired function $\varphi$ is obtained by regularizing $-\log v$ and a function similarly defined on $\{\varrho>0\}$.

Let us proceed to formulate a refined version of Proposition 1.1. Let $X, M$ and $\mathcal{F}$ be as in Proposition 1.1 and let $g$ be a Hermitian metric on $X$. For any Hermitian form $\Theta$ on the fibers of $T_{X}^{1,0}$, we shall denote by $\lambda_{p}(\Theta)(p=1, \ldots, n)$ the eigenvalues of $\Theta$ with respect to $g$ ordered in such a way that $\lambda_{1}(\Theta) \geq \cdots \geq \lambda_{n}(\Theta)$, where $n=\operatorname{dim} X$.

By $r(x)$ we shall denote the distance from $x$ to $M$ with respect to $g$. In view of the proof of Proposition 1.1, it is clear that the following is similarly true.

Proposition 1.2. Suppose that $N_{\mathcal{F}}$ admits a fiber metric whose curvature form is semipositive of rank $\geq k$ on the fibers of $T_{\mathcal{F}}^{1,0} \mid M$. Then there exists a real valued nonnegative $C^{\infty}$ function $\varphi$ on $X-M$ such that

$$
\begin{aligned}
& \varphi+\log r=O(1), \\
& \liminf _{x \rightarrow M} r(x)^{2} \lambda_{1}(\partial \bar{\partial} \varphi)>0, \\
& \liminf _{x \rightarrow M} \lambda_{p}(\partial \bar{\partial} \varphi)>0 \quad \text { for } 2 \leq p \leq k+1,
\end{aligned}
$$

and

$$
\liminf _{x \rightarrow M} \lambda_{p}(\partial \bar{\partial} \varphi) \geq 0 \quad \text { for } k+1<p \leq n .
$$

If $M$ is of class $C^{\infty}$, one may take the above $u_{\gamma}$ to be $C^{\infty}$, for which one can find a $C^{\infty}$ extension $\widetilde{u}_{\gamma}$, even defined on $W_{\gamma}$, not necessarily holomorphic on $W_{\gamma}-M$, but in such a way that $\bar{\partial} \widetilde{u}_{\gamma}$ vanishes to infinite order along $M$ (Whitney's theory). For this one does not need the existence of $\mathcal{F}$.

In the presence of such regularity, one can produce a function $\varphi$ satisfying (1.3)-(1.6) by imposing an appropriate positivity condition on the holomorphic normal bundle of $M$. Here the holomorphic normal bundle of $M$, say $N_{M}^{1,0}$, is defined as

$$
N_{M}^{1,0}:=\left(T_{X}^{1,0} \mid M\right) / \operatorname{Ker}\left(\partial \varrho \mid\left(T_{X}^{1,0} \mid M\right)\right)
$$

whose restriction to each $(n-1)$-dimensional complex submanifold of $M$ has a structure of holomorphic line bundle induced from that of $T_{X}^{1,0}$.

In fact, it is clear that one obtains the following by pointwise applying to $\widetilde{u}_{\gamma}$ the rest of the above mentioned construction of $\varphi$. 
Proposition 1.3. In the above situation, suppose that $N_{M}^{1,0}$ admits a fiber metric whose curvature form is everywhere semipositive of rank $\geq k$ on $\operatorname{Ker}\left(\partial \varrho\left|T_{X}^{1,0}\right| M\right)$. Then $X-M$ admits a function satisfying (1.3)-(1.6).

\section{§2. Basic facts on $L^{2}$ cohomology}

For the reader's convenience, we shall recall rudiments of the $L^{2}$ cohomology theory on complete Kähler manifolds. The goal here is, among other things, to present the statement of Theorem 4.1 of [Oh-Tg] (cf. Theorem 2.2 below). For the proof of Theorems 0.1 and 0.2 , Theorems 2.1 and 2.2 will be applied to $X-M$ equipped with a metric which is near $M$ locally asymptotically equivalent to the Bergman metric.

Let $\left(Y, g_{Y}\right)$ be a Hermitian manifold of dimension $n$ and let $(E, h)$ be a Hermitian holomorphic vector bundle over $Y$. We denote by $C^{p, q}(Y, E)$ the space of $E$-valued $C^{\infty}(p, q)$-forms on $Y . C_{0}^{p, q}(Y, E)$ will stand for the subset of $C^{p, q}(Y, E)$ consisting of compactly supported forms. The pointwise length of $u \in C^{p, q}(Y, E)$ is denoted by $|u|$. Then the $L^{2}$ norm of $u$, denoted by $\|u\|$, is defined as the square root of the integral of $|u|^{2}$ on $Y$ whenever $u \in C_{0}^{p, q}(Y, E)$.

Let $L^{p, q}(Y, E)$ be the completion of the pre-Hilbert space $C_{0}^{p, q}(Y, E)$ equipped with the $L^{2}$ norm. The operator $\bar{\partial}$, the complex exterior derivative of type $(0,1)$, will also stand for a densely defined closed linear operator on $L^{p, q}(Y, E)$ whose domain of definition, say Dom $\bar{\partial}$, is $\left\{f \in L^{p, q}(Y, E): \bar{\partial} f \in L^{p, q+1}(Y, E)\right\}$, where $\bar{\partial} f$ is defined in the sense of distributions.

We put

$$
\begin{aligned}
& H^{p, q}(Y, E):=\operatorname{Ker} \bar{\partial} \cap C^{p, q}(Y, E) / \operatorname{Im} \bar{\partial} \cap C^{p, q}(Y, E), \\
& H_{0}^{p, q}(Y, E):=\operatorname{Ker} \bar{\partial} \cap C_{0}^{p, q}(Y, E) / \operatorname{Im} \bar{\partial} \cap C_{0}^{p, q}(Y, E), \\
& H_{(2)}^{p, q}(Y, E):=\operatorname{Ker} \bar{\partial} \cap L^{p, q}(Y, E) / \operatorname{Im} \bar{\partial} \cap L^{p, q}(Y, E) .
\end{aligned}
$$

The bundle $E$ will not be indicated if $E=Y \times \mathbb{C}$, the trivial line bundle. The adjoint of $\bar{\partial}$ will be denoted by $\bar{\partial}^{*}$.

Proposition 2.1. If $\operatorname{Im} \bar{\partial} \cap L^{p, q}(Y, E)$ is closed, then

$$
H_{(2)}^{p, q}(Y, E) \cong \operatorname{Ker} \bar{\partial} \cap \operatorname{Ker} \bar{\partial}^{*} \cap L^{p, q}(Y, E)
$$

Proof. See $[\mathrm{K}]$ for instance.

The following variant of Grauert-Riemenschneider's vanishing theorem is essentially contained in $[\mathrm{Tg}]$ up to Serre duality. 
Theorem 2.1. Let $\left(Y, g_{Y}\right)$ be a connected complete Kähler manifold of dimension $n$, let $(L, h)$ be a Hermitian holomorphic line bundle over $Y$, and let $k$ be an integer such that $\sum_{p=k+1}^{n} \lambda_{p}\left(\Theta_{h}\right)$ is everywhere nonnegative and $\geq c$ for some positive constant $c$ outside a compact subset of $Y$. Then $H_{(2)}^{n, n-k}(Y, L)=\{0\}$ and $H_{(2)}^{0, k}\left(Y, L^{*}\right)=\{0\}$. Here $L^{*}$ denotes the dual bundle of $L$ equipped with the dual fiber metric.

Proof. By the curvature condition, there exists a compact set $K$ in $Y$ such that

$$
\int_{Y-K}|u|^{2} d V \leq\|\bar{\partial} u\|^{2}+\left\|\bar{\partial}^{*} u\right\|^{2}
$$

for any $u \in \operatorname{Dom} \bar{\partial} \cap \operatorname{Dom} \bar{\partial}^{*} \cap L^{n, n-k}(Y, L)$, where $d V$ denotes the volume form. (See [Oh-3] for instance.) From this estimate, it follows by Rellich's lemma and Hörmander's theorem that $\operatorname{Im} \bar{\partial} \cap L^{n, n-k}(Y, L)$ is closed and $\operatorname{dim} H_{(2)}^{n, n-k}(Y, L)$ $<\infty$ (cf. Theorem 1.1.3 in [H]). See also [Oh-1].

Hence $H_{(2)}^{n, n-k}(Y, L) \cong \operatorname{Ker} \bar{\partial} \cap \operatorname{Ker} \bar{\partial}^{*} \cap L^{n, n-k}(Y, L)$ by Proposition 2.1. By (2.4) it then follows that every element of $\operatorname{Ker} \bar{\partial} \cap \operatorname{Ker} \bar{\partial}^{*} \cap L^{n, n-k}(Y, L)$ is zero outside some compact subset of $Y$ so that it vanishes identically by Aronszajn's unique continuation theorem. Hence $H_{(2)}^{n, n-k}(Y, L)=\{0\}$. Similarly one obtains $H_{(2)}^{0, k}\left(Y, L^{*}\right)=\{0\}$.

Corollary 2.1. Let $\left(Y, g_{Y}\right)$ be as above and let $\varphi: Y \rightarrow[0, \infty)$ be a $C^{\infty}$ exhaustion function on $Y$ such that $\sum_{p=k+1}^{n} \lambda_{p}(\partial \bar{\partial} \varphi)$ is everywhere nonnegative and $\geq c$ for some positive constant $c$ outside a compact subset of $Y$. Then $H^{n, p}(Y)=\{0\}$ (resp. $\left.H_{0}^{0, p}(Y)=\{0\}\right)$ for $p \geq n-k$ (resp. $\left.p \leq k\right)$.

Proof. Let $u \in \operatorname{Ker} \bar{\partial} \cap C^{n, n-k}(Y)$. Then one can find a convex increasing function $\nu$ such that $u \in L^{n, n-k}(Y)$ with respect to the fiber metric $e^{-\nu(\varphi)}$. Hence, by Theorem 2.1 and the strong ellipticity of the complex Laplacian, one obtains $u \in$ $\operatorname{Im} \bar{\partial} \cap C^{n, n-k}(Y)$. Hence $H^{n, n-k}(Y)=\{0\}$. Clearly one has also $H^{n, p}(Y)=\{0\}$ for $p \geq n-k+1$. Hence, by the Serre duality one has $H_{0}^{0, p}(Y)=\{0\}$ for $p \leq k$.

Moreover, from the solvability of the $\bar{\partial}$-equation in the spaces $L^{0, p}(Y)$ with respect to the fiber metrics $e^{m \varphi}(m=1,2, \ldots)$, the following is inferred directly.

Corollary 2.2. Let $(X, g)$ be a compact Kähler manifold, let $M$ be a real hypersurface such that there exists a $C^{\infty}$ function $\varphi$ satisfying (1.3)-(1.6). Then, for any $m>0$ and $v \in \operatorname{Ker} \bar{\partial} \cap C^{0, p}(X)(p \leq k)$ satisfying $v=O\left(e^{-m \varphi}\right)$, there exists $a(0, p-1)$-form $u$ of class $C^{1}$ on $X$ such that $u \mid X-M \in C^{0, p-1}(X-M), \bar{\partial} u=v$ on $X$ and $u \mid M=0$. 
The main ingredient of [Oh-Tg], slightly strengthened for our purpose, is

Theorem 2.2. Let $\left(Y, g_{Y}\right)$ be a complete Hermitian manifold of dimension n, let $(E, h)$ be a Hermitian holomorphic vector bundle over $Y$ whose curvature form is zero outside a compact subset of $Y$, and let $k$ be a nonnegative integer. Suppose that $g_{Y}$ is Kählerian outside a compact subset and there exists an exhaustive $C^{\infty}$ function $\psi: Y \rightarrow \mathbb{R}$ such that

$$
\lim _{c \rightarrow \infty} \sup _{Y-Y_{c}} \gamma_{1}<l+1 / 100 n \text { and } \lim _{c \rightarrow \infty} \inf _{Y-Y_{c}} \gamma_{n-k+1}>l-1 / 100 n
$$

and

$$
\lim _{c \rightarrow \infty} \inf _{Y-Y_{c}} \lambda_{n}(\partial \bar{\partial} \psi-8 \partial \psi \wedge \bar{\partial} \psi) \geq-1 / 100 n
$$

where $\gamma_{1} \geq \cdots \geq \gamma_{n}$ are the eigenvalues of $\partial \bar{\partial} \psi$ and $Y_{c}:=\{x \in Y: \psi(x)<c\}$. If $p+q \geq n+k$ then $\operatorname{dim} H^{p, q}(Y, E)<\infty$ and $H^{p, q}(Y, E) \cong H_{(2)}^{p, q}(Y, E)$.

For the proof the reader is referred to [Oh-Tg]. In (2.5), the condition lim sup $\gamma_{1}<$ $l+1 / 100 n$ (resp. $\lim \inf \gamma_{n-k+1}>l-1 / 100 n$ ) is stated there as $\lim \sup \gamma_{1}=l$ (resp. $\liminf \gamma_{n-k+1}=l$ ), but (2.5) suffices in the proof (cf. (4.6) in [Oh-Tg]).

Corollary 2.3. Under the above situation, $\operatorname{dim} H_{0}^{p, q}(Y, E)<\infty$ and $H_{0}^{p, q}(Y, E) \cong$ $H_{(2)}^{p, q}(Y, E)$ both hold if $p+q \leq n-k$.

Recalling that the Lefschetz isomorphism exists between the $L^{2}$ harmonic forms of type $(p-s, q-s)$ and $(p, q)$ whenever $g_{Y}$ is Kähler and $h$ is flat, one infers the following from Theorem 2.2 and Corollary 2.3.

Corollary 2.4. Under the above situation, assume that $g$ is Kähler and $h$ is flat. Then the natural homomorphisms $H_{0}^{p, q}(Y, E) \rightarrow H_{(2)}^{p, q}(Y, E)$ and $H_{(2)}^{p, q}(Y, E) \rightarrow$ $H^{p, q}(Y, E)$ are surjective (resp. injective) if $p+q \geq n+k($ resp. $p+q \leq n-k)$.

In view of the exactness of the sequence

$$
H^{p, q-1}(Y, E) \rightarrow \underset{\lim _{K \subset Y}}{\rightarrow} H^{p, q-1}(Y-K, E \mid(Y-K)) \rightarrow H_{0}^{p, q}(Y, E) \rightarrow H^{p, q}(Y, E)
$$

where $K$ runs through the compact subsets of $Y$, one infers from Corollary 2.3 a Hartogs type extendibility:

Corollary 2.5. In the situation of Corollary 2.3, the restriction homomorphisms $H^{p, q}(Y, E) \rightarrow \lim _{K \subset Y} H^{p, q}(Y-K, E \mid(Y-K))$ are surjective for $p+q<n-k$.

Corollary 2.6. In the above situation, suppose that $Y=X-M$ for some compact complex manifold $X$ and a real hypersurface $M \subset X$, and that $E$ extends to 
a holomorphic vector bundle $E$ over $X$. Then the homomorphisms $H^{p, q}(X, E) \rightarrow$ $\lim _{b} H^{p, q}(U, E \mid U)$ are surjective for $p+q<n-k$. Here $U$ runs through the neighborhoods of $M$.

Similarly, one infers from Corollary 2.4 the following.

Corollary 2.7. In the situation of Corollary 2.2, for any $v \in \operatorname{Im} \bar{\partial} \cap C^{1,1}(X)$ satisfying $v \in L^{1,1}(X-M)$ with respect to a metric which coincides with $\varepsilon g+\delta \partial \bar{\partial} \varphi$ on $U-M$ for some neighborhood $U$ of $M$ and for sufficiently small $\varepsilon>0$ and $\delta>0$ with $\varepsilon \ll \delta$, there exists $a u \in L^{1,0}(X-M)$ such that $\bar{\partial} u=v$ on $X-M$.

Note that, if $v=\bar{\partial} f$ for some $C^{\infty}(1,0)$-form $f$ on $X, f-u$ becomes a holomorphic 1-form on $X$ which coincides with $f$ on $M$, because of the asymptotic behavior of the volume form of $\varepsilon g+\delta \partial \bar{\partial} \varphi$ along $M$.

\section{§3. Proof of Theorem 0.1}

Let $X, M$ and $\mathcal{F}$ be as in the hypothesis of Theorem 0.1, let $g$ be a Kähler metric on $X$, and let $\varphi$ be as in Proposition 1.2 for $k=2$. Then, for any sufficiently small $\varepsilon>0$, the Hermitian form $g+\varepsilon \partial \bar{\partial} \varphi$ becomes a complete Kähler metric on $X-M$ in such a way that the hypothesis on $\partial \bar{\partial} \varphi$ in Corollary 2.1 is satisfied with respect to $g+\varepsilon \partial \bar{\partial} \varphi$.

Hence the bundle $N_{\mathcal{F}}$ extends from a neighborhood of $M$ to $X$ as a topologically trivial holomorphic line bundle, say $\widetilde{N}$. Since $X$ is a compact Kähler manifold, $\widetilde{N}$ is flat.

By Corollary 2.6, any defining 1-form $\omega$ of $\mathcal{F}$ on a connected neighborhood of $M$ is analytically extended to $X$ as an $\widetilde{N}$-valued holomorphic 1-form, say $\omega$. Since $\widetilde{N}$ is (unitarily) flat, $\omega$ is $d$-closed. This means that the leaves of $\mathcal{F}$ that are disjoint from $M$ stay away from $M$ forever. Namely, the distance, with respect to $g$, from a leaf, say $F$, of $\mathcal{F}$ to $M$ is positive whenever $F \cap M=\emptyset$ (cf. [Oh-5] or [Oh-7]). This clearly means the existence of a leaf $F^{\prime}$ such that $\varphi \mid F^{\prime}$ attains its maximum, which contradicts the property of the Levi form of $\varphi$.

Remark. The above proof in the spirit of [Oh-5] shows in particular that what really leads to a contradiction is the existence of a Kähler metric on $X$, the foliation $\mathcal{F}$ and an exhaustion function $\varphi$ on $X-M$ as above. However, it must be noted that the curvature assumption on $N_{\mathcal{F}}$, combined with $H_{0}^{0,2}(X-M)=0$, leads more directly to a contradiction as shown by Brunella [B-1]. Namely, the curvature form of $N_{\mathcal{F}}$ then extends to $X$ as a closed form which turns out to be $\partial \bar{\partial}$-exact by the Kähler assumption. This contradicts the maximum principle on $M$. In this shorter proof, the curvature assumption is used in two different places. 


\section{$\S 4$. Proof of Theorem 0.2}

Let $\varphi$ be a function satisfying (1.3)-(1.6) guaranteed by Proposition 1.3. Then, similarly to the proof of Theorem 0.1 , by applying Corollary 2.2 instead of Corollary 2.1, the holomorphic normal bundle of $M$ is extended to $X$ as a flat line bundle, say $N$. Then, exploiting the injectivity of $H_{(2)}^{1,1}(X-M, N) \rightarrow H^{1,1}(X-M, N)$ with respect to $\varepsilon g+\delta \partial \bar{\partial} \varphi$ as in Corollary 2.7, one can extend any $C^{\infty}$ defining 1 -form of the foliation on $M$ to an $N$-valued holomorphic 1-form, say $\omega$, on $X$. Since $\omega$ defines a foliation on a neighborhood of $M$, the rest of the argument is similar to that for Theorem 0.1. (See also [Oh-5, §3], and the remark in $\S 3$ above.)

\section{Acknowledgements}

The author is very grateful to the referee for careful reading and useful comments.

\section{References}

[A] R. A. Airapetian, Extending CR functions from piecewise smooth CR manifolds, Math., Sb. 134 (1987), 108-118 (in Russian). Zbl 0635.32012 MR 0912414

[B-1] M. Brunella, On the dynamics of codimension one holomorphic foliations with ample normal bundle, Indiana Univ. Math. J. 57 (2008), 3101-3113. Z Zbl 1170.37023 MR 2492227

[B-2] Codimension one foliations on complex tori, Ann. Fac. Sci. Toulouse Math. (6) 19 (2010), 405-418. Zbl 1246.53035 MR 2674768

[B-3] On Kähler surfaces with semipositive Ricci curvature, Riv. Math. Univ. Parma (N.S.) 1 (2010), 441-450. Zbl 1226.32011 MR 2789451

[C-L] D. Cerveau and A. Lins-Neto, Local Levi-flat hypersurfaces invariants by a codimension one holomorphic foliation, Amer. J. Math. 133 (2011), 677-716. Zbl 1225.32038 MR 2808329

[Dm] J.-P. Demailly, Cohomology of $q$-convex spaces in top degrees, Math. Z. 204 (1990), 283-295. Zbl 0682.32017 MR 1055992

[E] G. Elencwajg, Pseudo-convexité locale dans les variétés kählériennes, Ann. Inst. Fourier (Grenoble) 25 (1975), no. 2, 295-314. Zbl 0278.32015 MR 0387662

[F-Oh] H. Fuse and T. Ohsawa, On a curvature property of effective divisors and its application to sheaf cohomology, Publ. RIMS Kyoto Univ. 45 (2009), 1033-1039. Zbl 1190.32009 MR 2597128

[H] L. Hörmander, $L^{2}$ estimates and existence theorems for the $\bar{\partial}$ operator, Acta Math. 113 (1965), 89-152. Zbl 0158.11002 MR 0179443

[K] K. Kodaira, Harmonic fields in Riemannian manifolds (generalized potential theory), Ann. of Math. 50 (1949), 587-665. Z Zbl 0034.20502 MR 0031148

[L] A. Lins-Neto, A note on projective Levi flats and minimal sets of algebraic foliations, Ann. Inst. Fourier (Grenoble) 49 (1999), 1369-1385. Zbl 0963.32022 MR 1703092

[M] K. Matsumoto, Representation and non-degeneracy condition for Levi form of distance to real hypersurfaces in $\mathbb{C}^{n}$, Kyushu J. Math. 63 (2009), 291-300. Zbl 1187.32008 MR 2568774 
[Oh-1] T. Ohsawa, Finiteness theorems on weakly 1-complete manifolds, Publ. RIMS Kyoto Univ. 15 (1979), 853-870. Zbl 0434.32014 MR 0566085

[Oh-2] _ A reduction theorem for cohomology groups of very strongly $q$-convex Kähler manifolds, Invent. Math. 63 (1981), 335-354. Zbl 0457.32007 MR 0662598

[Oh-3] Isomorphism theorems for cohomology groups of weakly 1-complete manifolds, Publ. RIMS Kyoto Univ. 18 (1982), 191-232. Zbl 0526.32016 MR 0660827

[Oh-4] Pseudoconvex domains in $\mathbb{P}^{n}$ : a question on the 1-convex boundary points, in Analysis and geometry in several complex variables (Katata, 1997), Trends Math., Birkhäuser, 1999, 239-252. Zbl 0971.32007 MR 1699848

[Oh-5] , On the complement of Levi-flats in Kähler manifolds of dimension $\geq 3$, Nagoya Math. J. 185 (2007), 161-169. Zbl 1141.32015 MR 2301464

[Oh-6] A r eduction theorem for stable sets of holomorphic foliations on complex tori, Nagoya Math. J. 195 (2009), 41-56. Zbl 1187.32009 MR 2552952

[Oh-7] L Levi flat hypersurfaces in complex manifolds, in Complex analysis and digital geometry, Acta Univ. Upsaliensis Skr. Uppsala Univ. C Organ. Hist. 86, Uppsala Universitet, Uppsala, 2009, 223-231. Zbl 1213.32023 MR 2742682

[Oh-Tg] T. Ohsawa and K. Takegoshi, Hodge spectral sequence on pseudoconvex domains, Math. Z. 197 (1988), 1-12. Zbl 0638.32016 MR 0917846

[O] K. Oka, Collected papers, Springer, 1984. Zbl 0541.01013 MR 0754337

[P] Ch. Pommerenke, Boundary behaviour of conformal maps, Grundlehren Math. Wiss. 299, Springer, Berlin, 1992. Zbl 0762.30001 MR 1217706

[Sh] N. V. Shcherbina, Decomposition of a common boundary of two domains of holomorphy into analytic curves, Izv. Akad. Nauk SSSR Ser. Mat. 46 (1982), 1106-1123 (in Russian). Zbl 0518.32008 MR 0675533

[S] Y.-T. Siu, Nonexistence of smooth Levi-flat hypersurfaces in complex projective spaces of dimension $\geq 3$, Ann. of Math. 151 (2000), 1217-1243. Zbl 0980.53065 MR 1779568

[Tg] K. Takegoshi, A generalization of vanishing theorems for weakly 1-complete manifolds, Publ. RIMS Kyoto Univ. 17 (1981), 311-330. Z Zbl 0464.32017 MR 0613949

[T-1] A. Takeuchi, Domaines pseudoconvexes infinis et la métrique riemannienne dans un espace projectif, J. Math. Soc. Japan 16 (1964), 159-181. Zbl 0141.08804 MR 0173789

[T-2] _ Domaines pseudoconvexes sur les variétés kählériennes, J. Math. Kyoto Univ. 6 (1967), 323-357. Zbl 0179.12203 MR 0217335 